\title{
The durability of endoscopic third ventriculostomy and ventriculoperitoneal shunts in children with hydrocephalus following posterior fossa tumor resection: a systematic review and time-to-failure analysis
}

\author{
Michael C. Dewan, MD, Jaims Lim, BS, Chevis N. Shannon, MBA, MPH, DrPH, and \\ John C. Wellons III, MD, MSPH
}

Division of Pediatric Neurosurgery, Department of Neurological Surgery, Vanderbilt University Medical School, Nashville, Tennessee

OBJECTIVE Up to one-third of patients with a posterior fossa brain tumor (PFBT) will experience persistent hydrocephalus mandating permanent CSF diversion. The optimal hydrocephalus treatment modality is unknown; the authors sought to compare the durability between endoscopic third ventriculostomy (ETV) and ventriculoperitoneal shunt (VPS) therapy in the pediatric population.

METHODS The authors conducted a systematic review of articles indexed in PubMed between 1986 and 2016 describing ETV and/or VPS treatment success/failure and time-to-failure rate in patients < 19 years of age with hydrocephalus related to a PFBT. Additionally, the authors conducted a retrospective review of their institutional series of PFBT patients requiring CSF diversion. Patient data from the systematic review and from the institutional series were aggregated and a time-to-failure analysis was performed comparing ETV and VPS using the Kaplan-Meier method.

RESULTS A total of 408 patients were included from 12 studies and the authors' institutional series: 284 who underwent ETV and 124 who underwent VPS placement. The analysis included uncontrolled studies with variable method and timing of CSF diversion and were subject to surgeon bias. No significant differences between cohorts were observed with regard to age, sex, tumor grade or histology, metastatic status, or extent of resection. The cumulative failure rate of ETV was $21 \%$, whereas that of VPS surgery was $29 \%(p=0.105)$. The median time to failure was earlier for ETV than for VPS surgery (0.82 [IQR 0.2-1.8] vs 4.7 months [IQR 0.3-5.7], $p=0.03)$. Initially the ETV survival curve dropped sharply and then stabilized around 2 months. The VPS curve fell gradually but eventually crossed below the ETV curve at 5.7 months. Overall, a significant survival advantage was not demonstrated for one procedure over the other $(p=0.21, \log$ rank). However, postoperative complications were higher following VPS (31\%) than ETV $(17 \%)(p=0.012)$.

CONCLUSIONS ETV failure occurred sooner than VPS failure, but long-term treatment durability may be higher for ETV. Complications occurred more commonly with VPS than with ETV. Limited clinical conclusions are drawn using this methodology; the optimal treatment for PFBT-related hydrocephalus warrants investigation through prospective studies.

https://thejns.org/doi/abs/10.3171/2017.1.PEDS16536

KEY WORDS durability; endoscopic third ventriculostomy; hydrocephalus; pediatric; posterior fossa; tumor; ventriculoperitoneal shunt

$\mathrm{B}$ ETWEEN one-quarter and one-third of patients with a posterior fossa brain tumor (PFBT) will experience persistent hydrocephalus following posterior fossa resection. $3,7,11,21,27,29$ Historically, this comorbidity has been addressed by placement of a ventriculoperitoneal shunt (VPS), the complications and failures of which are well described. 4,27,28,30 Within the last 2 decades, the utility of endoscopic third ventriculostomy (ETV) for this etiology of hydrocephalus has been demonstrated by long-term success rates that, in some reports, eclipse those of VPS surgery. ${ }^{20,26,29}$ Most studies, however, are plagued by limited patient numbers, which restricts power, and uncontrolled

ABBREVIATIONS EOR = extent of resection; ETV = endoscopic third ventriculostomy; PFBT = posterior fossa brain tumor; VPS = ventriculoperitoneal shunt. SUBMITTED September 18, 2016. ACCEPTED January 12, 2017.

INCLUDE WHEN CITING Published online March 10, 2017; DOI: 10.3171/2017.1.PEDS16536. 
study design, which injects bias. The relative effectiveness between ETV and VPS therapy in patients with persistent hydrocephalus is unknown. ${ }^{18}$ In this study, we conducted a systematic review of the durability of treatment for PFBTrelated hydrocephalus and compared the time to reintervention for ETV and VPS.

\section{Methods}

\section{Article Selection}

A PubMed search was performed for articles published between 1986 and 2016 in English using the following search algorithm: (((pediatrics OR pediatrics OR pediatric) OR (child OR child OR children)) AND (hydrocephalus OR hydrocephalus)) AND (tumor OR neoplasms OR neoplasms OR tumor)) AND (ETV OR shunt OR (CSF AND diversion)) AND (infant OR child OR adolescent). Initially, 3518 article titles were reviewed for content and relevance. References from reviewed articles were also examined for possible incorporation. Articles were included if they contained the following data: 1) incidence and timing of shunt and/or ETV failure, 2) pediatric patients $(<19$ years) with PFBT-associated hydrocephalus, and 3) distinguishable failure rates between either primary VPS surgery or ETV. Exceptions were made for studies without both incidence and timing of failure, if at least one (incidence or timing) was reported and procedure-specific details regarding at least 4 of the covariates listed in Table 1 were reported. Such studies were included to maximize the integrity of characterization of the 2 treatment cohorts and thereby promote generalizability to nonstudy cohorts of ETV or VPS therapy. Treatment failure was designated whenever a repeat intervention for hydrocephalus was reported, including repeat ETV or VPS insertion (for primary ETV), or VPS revision or ETV (for a primary VPS). Complications from each source article were tabulated across studies and included infection, shunt malposition, aborted ETV, ependymal vascular injury, extraaxial hemorrhagic or CSF collection, ocular palsy, and hypothalamic disturbance. Studies were excluded for any of the following reasons: adult and pediatric patients were indistinguishable, PFBTs were indistinguishable from other tumor types/ locations, and intrinsic brainstem gliomas and/or pineal region tumors were present. Only peer-reviewed articles were considered for inclusion; abstracts and presentations were ineligible. The rationale of article inclusion is depicted in the PRISMA flow diagram ${ }^{19}$ (Fig. 1).

\section{Original Data Extraction}

Given the imbalanced treatment groups caused by a paucity of published data on shunt durability, original data were extracted from our institutional PFBT database, which included 151 posterior PFBT resections between 2002 and 2015. Because of the surgical preference by a former institutional surgeon, 33 patients with persistent postoperative hydrocephalus were treated using VPS - a decision that was independent of baseline patient and tumor characteristics. No institutional patients were offered ETV. Patient demographics, tumor characteristics, resection data, and hydrocephalus treatment and outcome were
TABLE 1. Studies included in the systematic review

\begin{tabular}{lrrr}
\hline & \multicolumn{3}{c}{ No. of Patients/Cohort } \\
\cline { 2 - 4 } \multicolumn{1}{c}{ Authors \& Year } & Total $^{*}$ & ETV & VPS \\
\hline Schneider et al., 2015 & 28 & 5 & 23 \\
\hline Azab et al., 2013 & 17 & 17 & 0 \\
\hline El-Ghandour, 2011 & 53 & 32 & 21 \\
\hline Roujeau et al., 2011 & 11 & 2 & 9 \\
\hline El Beltagy et al., 2010 & 40 & 40 & 0 \\
\hline Bhatia et al., 2009 & 40 & 36 & 4 \\
\hline Depreitere et al., 2007 & 26 & 14 & 12 \\
\hline Klimo \& Goumnerova, 2006 & 2 & 2 & 0 \\
\hline Ray et al., 2005 & 27 & 27 & 0 \\
\hline Ruggiero et al., 2004 & 30 & 24 & 6 \\
\hline Grunert et al., 2003 & 10 & 10 & 0 \\
\hline Sainte-Rose et al., 2001 & 91 & 75 & 16 \\
\hline Vanderbilt cohort† & 33 & 0 & 33 \\
\hline Total & 408 & 284 & 124 \\
\hline
\end{tabular}

* Numbers represent the total number meeting criteria for the review, not necessarily the total number of patients within the original article.

$\dagger$ Vanderbilt cohort represents the results of a retrospective review of patients treated for PFBT-related hydrocephalus between 2002 and 2015.

documented and incorporated along with the systematic review data. For extraction of institutional data, IRB approval was obtained.

\section{Statistical Analysis}

Descriptive data are reported as the mean (SD) or median (IQR) as dictated by their distribution, with relative proportions as percentages. Nonnormal continuous data were compared using the Wilcoxon rank-sum test. Categorical variables, including the presence of failure between groups, was compared via the chi-square test. Survival curves were created utilizing the Kaplan-Meier method, with the time-to-first-failure data as the end point of interest. Here, survival is defined as the absence of need for a subsequent CSF-diverting procedure and absence of death from hydrocephalus. Unadjusted equality of survival duration was examined with the log-rank test. A multivariate Cox regression was not performed given the nature of the pooled data, which was not disaggregated for each covariate relative to the individual patient.

Data were initially collected and managed using Research Electronic Data Capture (REDCap) tools. ${ }^{14}$ Data analysis was conducted utilizing Stata v14 (StataCorp LLC).

\section{Results}

In total, 12 studies were included., 1,2,6,9,10,13,15,22,24-27 Figure 1 demonstrates stepwise exclusion of candidate articles, following PRISMA guidelines. ${ }^{19}$ Along with 33 VPS patients from Vanderbilt, a total of 408 patients were included within the analysis: 284 who underwent ETV and 124 who underwent VPS insertion. All studies were retrospective cohort reviews of institutional data, and all were published after 2000. Expectedly, the distribution 


\section{Initial PubMed search}

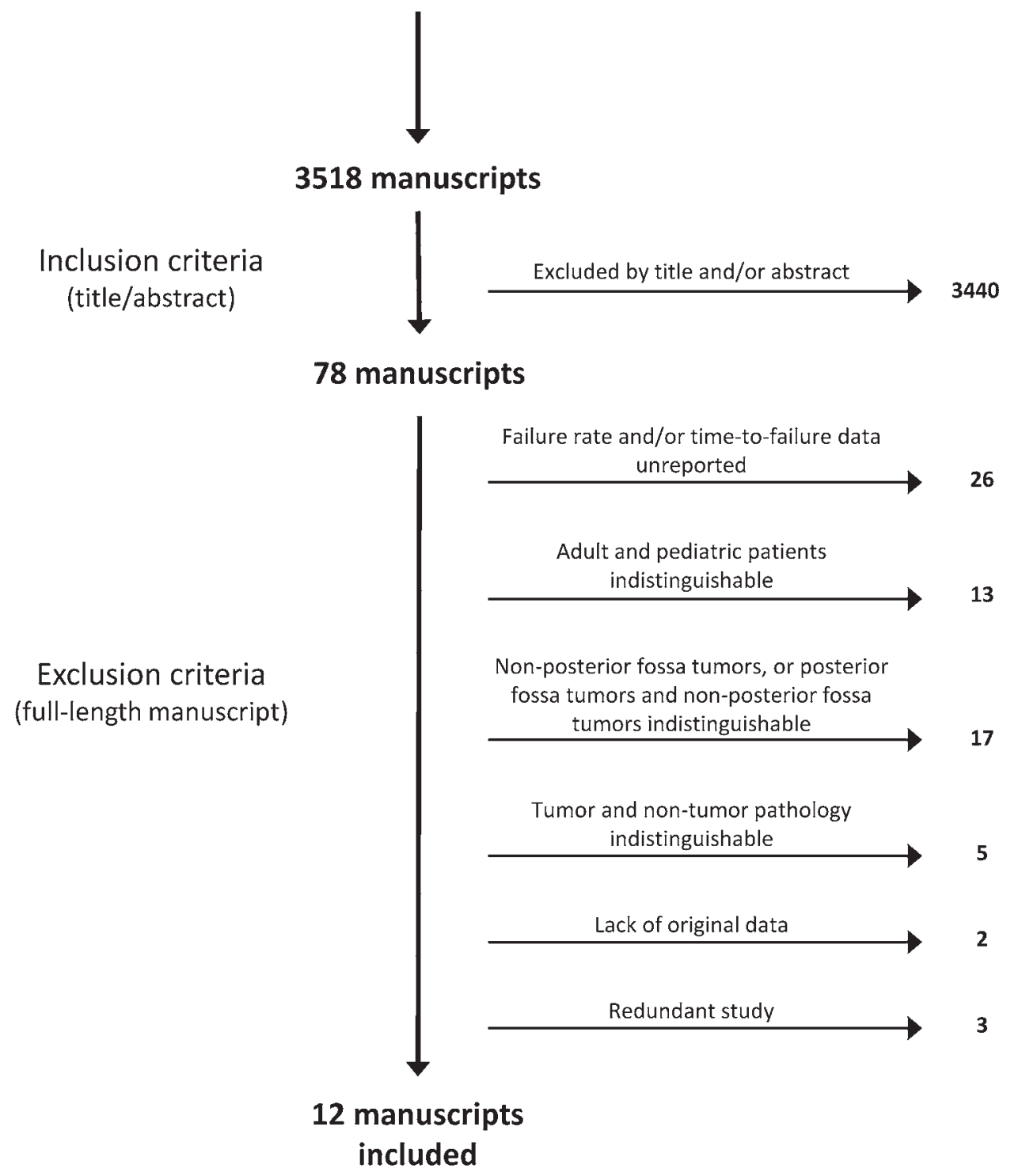

FIG. 1. PRISMA flow diagram. The stepwise progression from search term inquiry to final article inclusion is outlined. A total of 12 studies were selected from more than 3500 articles.

between ETV and VPS surgeries across studies was heterogeneous; treatment allocation within each study is displayed in Table 1.

Comparing ETV and VPS groups, there were no significant intergroup differences in age, sex, tumor grade or histology, metastatic status, or extent of resection (EOR). Full patient characteristics are listed in Table 2. As a result of incomplete data within included papers, the sum of tabulations from some categorical counts do not equal the full cohort.

The median times to last follow-up for the ETV and VPS groups were 26.2 months (IQR 21.0-27.4 months) and 25 months (IQR 22.8-37 months), respectively. The cumulative failure rate of ETV was $21 \%$, whereas that of VPS was 29\%, but this difference did not reach statistical significance $(\mathrm{p}=0.11)$. The median time to failure was earlier for ETV than for VPS (0.82 months [IQR 0.2-1.8 months] vs 4.7 months [IQR 0.3-5.7 months], $\mathrm{p}=0.03$ ). The Kaplan-Meier survival curves in Fig. 2 depict the relative durability of CSF diversion between ETV and VPS surgery. Early ETV failures produced a steep curve that leveled beyond 2 months. The VPS curve, on the other hand, was more gradual initially and crossed the ETV curve at 171 days, or 5.7 months. While fine differences between curves can be appreciated visually, a significant survival advantage was not demonstrated for one procedure over the other ( $\mathrm{p}=0.21, \log$-rank, unadjusted).

Postoperative complications were higher following VPS placement $(31 \%)$ than ETV $(17 \%)(\mathrm{p}=0.012)$ and included infections, shunt malposition, extraaxial hemorrhages, and cranial nerve palsies (variably detailed among source articles). A single death was reported in a case 
TABLE 2. Baseline patient data stratified by hydrocephalus procedure

\begin{tabular}{|c|c|c|c|c|}
\hline \multirow[b]{2}{*}{ Characteristic } & \multicolumn{3}{|c|}{ No. of Patients/Cohort } & \multirow[b]{2}{*}{ p Value } \\
\hline & Overall $(n=408)$ & $\operatorname{ETV}(n=284)$ & $\operatorname{VPS}(n=124)$ & \\
\hline \multicolumn{5}{|l|}{ Age in mos } \\
\hline Median (IQR) & $79.2(72-86.4)$ & $79.2(78-84)$ & $84(59-86.4)$ & 0.546 \\
\hline Mean \pm SD & $80.29 \pm 28.36$ & $81.7 \pm 26.2$ & $77.8 \pm 31.7$ & 0.876 \\
\hline Male (\%) & $84(59.1)$ & $35(51.5)$ & $49(66.2)$ & 0.074 \\
\hline Grade (\%) & & & & 0.781 \\
\hline High & $158(74.5)$ & $105(73.9)$ & $53(75.7)$ & \\
\hline Low & $54(25.5)$ & $37(26.1)$ & $17(24.3)$ & \\
\hline Histology (\%) & & & & 0.382 \\
\hline Astrocytoma & $68(24.7)$ & $43(23.4)$ & $25(27.5)$ & \\
\hline Medulloblastoma & $136(49.5)$ & $88(47.8)$ & $48(52.8)$ & \\
\hline Ependymoma & $64(23.3)$ & $47(25.5)$ & $17(18.7)$ & \\
\hline Others & $7(2.6)$ & $6(2.6)$ & $1(1.1)$ & \\
\hline Cerebral metastases (\%) & $26(25)$ & $18(24.7)$ & $8(25.8)$ & 0.901 \\
\hline EOR (\%) & & & & 0.332 \\
\hline GTR & $131(56.2)$ & $101(57.4)$ & $30(52.6)$ & \\
\hline STR & $54(23.2)$ & $49(27.8)$ & $5(8.8)$ & \\
\hline Debulking & $6(2.6)$ & $5(2.8)$ & $1(1.8)$ & \\
\hline Biopsy & $29(12.5)$ & $17(9.7)$ & $12(21.1)$ & \\
\hline \multicolumn{5}{|l|}{ Procedural details (\%) } \\
\hline Complications & $57(20.8)$ & $35(17.2)$ & $22(31.4)$ & 0.012 \\
\hline Death & $1(0.2)$ & $0(0)$ & $1(1.6)$ & 0.08 \\
\hline Failure (\%) & $89(23)$ & $60(21.1)$ & $29(29.0)$ & 0.105 \\
\hline
\end{tabular}

GTR = gross-total resection; STR = subtotal resection.

in which ventriculitis developed and the patient died 4.5 months after VPS insertion.

\section{Discussion}

In this systematic review, data were pooled from studies reporting the rate and timing of failure of CSF diversion between ETV and VPS. With more than 400 patients, this represents the largest analysis examining the relative durability between ETV and VPS in patients treated for hydrocephalus secondary to a PFBT. Overall, the 2 cohorts were well matched in terms of age, tumor characteristics, and EOR, lending validity to subsequent comparison analyses. Furthermore, the median age, the pattern of specific tumor grades requiring CSF diversion, and the distribution of EOR all compare favorably with the literature. ${ }^{11,23,25,26,30}$ There was no significant difference in overall failure rate or in survival duration between the 2 CSF-diversion groups. The burden of failures occurred sooner for ETV than for VPS surgery, while more postoperative complications were observed following VPS treatment than ETV.

Hydrocephalus in the setting of a PFBT is common, occurring in approximately $80 \%$ of patients before resection..$^{5,30}$ While the majority will experience resolution following removal of the obstructing pathology, about $30 \%$ will have persistent hydrocephalus requiring permanent CSF diversion. .,7,21,29 Riva-Cambrin et al. have developed, and then modified, a clinical score to help surgeons predict which patients are likely to develop postoperative hydrocephalus, based upon age, degree of hydrocephalus, presence of metastases, presence of transependymal edema, and preresection estimated tumor diagnosis. ${ }^{11,23}$ Patients with a low likelihood of persistent hydrocephalus could be spared prophylactic, extraneous CSF diversion. But while the modified Canadian Preoperative Prediction Rule for Hydrocephalus (or mCPPRH) scale may help determine if a patient will need CSF diversion, it remains unclear how such a patient is best treated-ETV or VPS?

There are very few studies specifically reporting duration of VPS survival, likely a product of publication bias. In the largest such study, incorporating more than 3500 patients, Cochrane and Kestle reported a 6-month cumulative success rate of approximately $65 \% .{ }^{5}$ However, this included patients from within all origins of hydrocephalus, and the study itself was designed to detect differences between surgeon experience levels. In a separate study including 28 patients with persistent hydrocephalus following medulloblastoma resection, no patients with a VPS required early reintervention (100\% success) compared with 4 of 5 ETV patients in whom the ETV was converted to a VPS (20\% success). ${ }^{27}$

More recently, however, ETV has been advocated given its familiarity among pediatric neurosurgeons and the inherent freedom from hardware dependence. Both Drake ${ }^{8}$ and Kulkarni et al. ${ }^{17}$ reported that ETV was successful in more than two-thirds of the ETV-treated patients among 


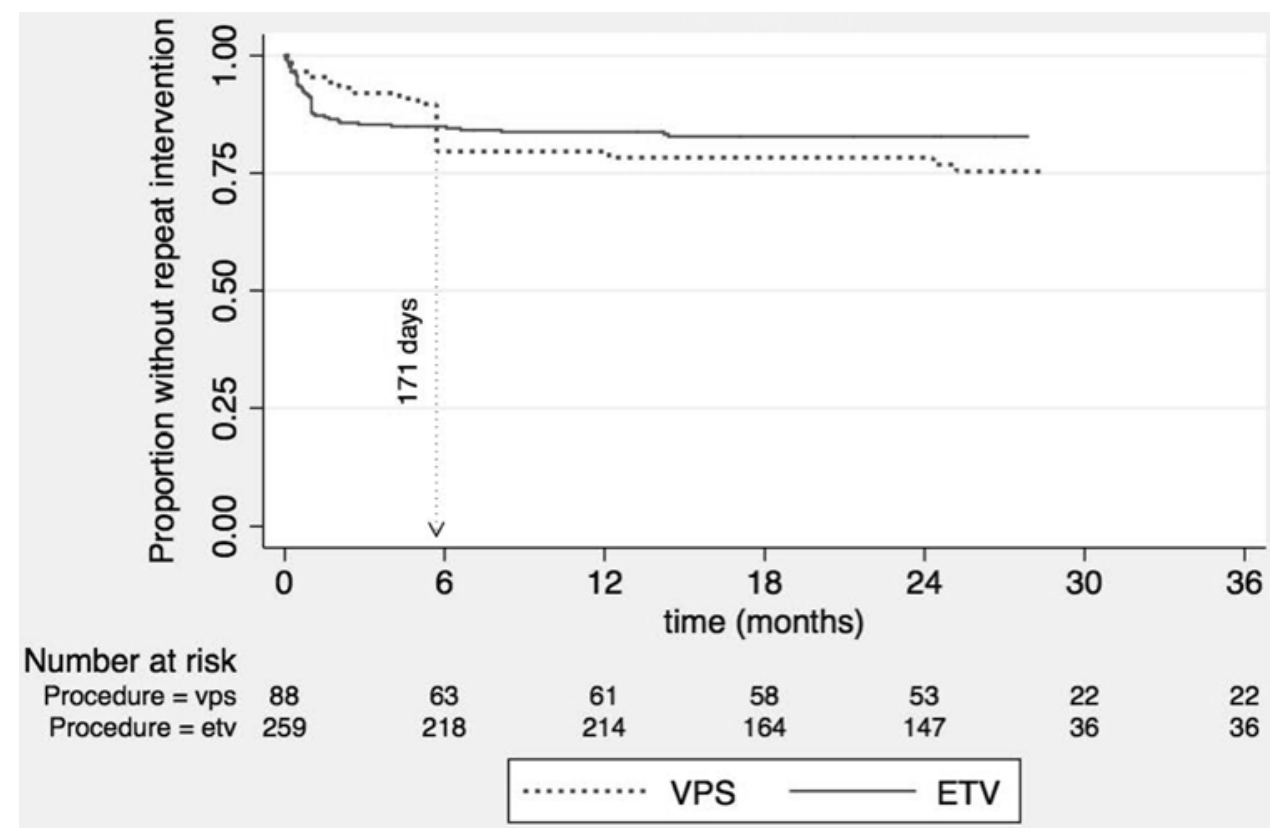

FIG. 2. Kaplan-Meier survival curves for ETV versus VPS. The time to reintervention for first-time CSF-diversionary ETV versus VPS surgery is compared in patients with PFBT-related hydrocephalus. The vertical arrow at 171 days represents the time at which the cumulative VPS survival curve crossed below that for ETV. A statistically significant survival advantage between the cohorts was not observed ( $p=0.205$, log-rank test).

those with tumor-related hydrocephalus. Others studies with more modest enrollment figures have reported success rates exceeding $90 \% .^{20,29}$

In the largest study to date comparing these 2 treatments, Kulkarni et al. analyzed more than 1200 patients with hydrocephalus, 223 (18\%) of whom the etiology was tumor related. ${ }^{16}$ Our results reflect their findings on several fronts. First, there appears to be a higher risk of early failure among patients treated with ETV. Specifically, Kulkarni et al. found a 20\% higher early failure rate in the ETV group relative to the VPS group. Second, over time, the risk of ETV failure became lower than that of VPS failure, indicating that the treatment advantage of ETV is only seen beyond the early postoperative period. Third, the point at which the unadjusted survival curves overlap (i.e., the time point at which the risk of VPS failure supersedes that of ETV) occurs at or before 6 months postoperatively: at 3 months in the Kulkarni et al. report ${ }^{16}$ and at 5.7 months in our pooled analysis.

The implications of these results on a potential trial comparing ETV and VPS surgery for PFBT-related hydrocephalus warrant comment. While a statistically significant difference in failure rates (29\% vs $21 \%$ ) was not observed, this likely represents cohort imbalance, rather than true power limitation. Assuming a power of 0.8 and Type I error of $0.05,370$ patients would need to be enrolled in such a trial. Collaborative clinical trial networks such as the Hydrocephalus Clinical Research Network or the Children's Oncology Group could design and conduct a trial spanning 3-5 years. Beyond simply informing the field of the absolute difference in success between procedures, such a study would also elucidate potential predictors of failure in PFBT-related hydrocephalus, including age, prior CSF-diversion procedure, and/or radiologically depicted cisternal architecture.

\section{Limitations, Perspective, and Future Directions}

The findings of this study must be carefully examined in relation to several important limitations. First, no study included controlled treatment cohorts, few described a balanced treatment allocation, some reported variable timing of ETV, and several reported findings from only a single treatment arm. As a result, for each study, surgeon bias must be considered, and balanced characteristics between ETV and VPS cohorts, while suggested, cannot be confirmed. Many well-designed, impactful studies on hydrocephalus related to PFBT had to be excluded, because details of either treatment failure or time to failure were not reported among the results. ${ }^{8,17,20,23,29}$ Additionally, because individualized patient variables could not be extracted from most articles, multivariate analysis was not possible; therefore, survival figures were not adjusted for important known predictors of ETV/VPS failure, such as age. Instead, we could only demonstrate overall equality between ETV and VPS cohorts as a whole (Table 2). Also, due to a lack of data reporting within articles, we were unable to describe the number of revision or redo surgeries performed over the follow-up period. This is a particularly relevant limitation when considering patient inconvenience and resource utilization between 2 procedures. Anatomical and clinical variations such as ventricular volume, adjuvant chemotherapy or radiotherapy, and disposition status, are also important variables that were excluded from our analysis secondary to mixed reporting. Similarly, limited and heterogeneous reporting of compli- 
cations precluded a detailed comparison of the type and degree of complications between cohorts. Last, this study was unable to examine the role of perioperative external ventricular drainage and its potential influence on ETV or VPS durability.

In light of these limitations, it is our opinion that the durability values between ETV and VPS carry more value as descriptive entities recounting the available literature, rather than conclusive evidence to support or refute a previously held bias regarding the optimal method of CSF diversion in PFBT. At best, the absence of a survival advantage between the 2 surgeries in this study provides equipoise on the subject matter. To this end, these results should serve less as definitive evidence and more as a call to action to the pediatric neurosurgery community. Currently there is no high- or moderate-level evidence offering insight into the durability of ETV and VPS for PFBT. And yet tumor-related obstructive hydrocephalus is among the most common causes of hydrocephalus in the pediatric population. ${ }^{8,17}$ Moreover, while its prevalence alone should motivate a search for answers, the intrinsic survival-limiting nature of the disease makes the question of treatment durability even more crucial. For example, the relative impact of a repeat ETV on a patient with medulloblastoma awaiting craniospinal irradiation is perhaps greater than for a child with longstanding congenital hydrocephalus. Similarly, multiple VPS revision surgeries following ependymoma resection might more dramatically influence the remaining quality of life for a child who has been given a limited 5-year survival prognosis than for a child with hydrocephalus not caused by a high-grade brain tumor. Whether the child has had an ETV or a VPS operation, complication-free durability-especially for the first 2-3 years-is critical for this population.

Many studies have examined predictors of hydrocephalus following PFBT resection. ${ }^{11,12,23}$ These authors have eloquently described the relative contribution of several variables to the development of hydrocephalus, and they have provided invaluable information for surgical decision making and patient and family counseling. But how are we to proceed after hydrocephalus has been diagnosed? Which approach will minimize future hydrocephalus interventions while still providing adequate CSF diversion? Perhaps there is more than one answer. These and others' results suggest that a VPS system is less susceptible to very early failure but that over time ETV may be more durable. Is it possible, then, that VPS is the best option for children with aggressive tumors and limited survival, while those with low-grade lesions fare better with ETV? Issues like these remain uncertain, yet, with greater clarity, may too come improvements in quality of life, resource utilization, and overall survival. Between multiinstitutional collaboration and thoughtful study design, it seems the tools to answer these questions are well within reach.

\section{Conclusions}

Endoscopic third ventriculostomy failure occurs sooner than VPS failure, but the long-term treatment durability may be higher in the former. Complications occur more commonly following VPS than ETV. The optimal treat- ment for PFBT-related hydrocephalus warrants investigation through prospective studies.

\section{Acknowledgments}

Grant funding support for this study was received from the National Center for Advancing Translational Sciences/National Institutes of Health (grant no. UL1 TR000445) for Vanderbilt REDCap.

\section{References}

1. Azab W, Al-Sheikh T, Yahia A: Preoperative endoscopic third ventriculostomy in children with posterior fossa tumors: an institution experience. Turk Neurosurg 23:359-365, 2013

2. Bhatia R, Tahir M, Chandler CL: The management of hydrocephalus in children with posterior fossa tumours: the role of pre-resectional endoscopic third ventriculostomy. Pediatr Neurosurg 45:186-191, 2009

3. Bognár L, Borgulya G, Benke P, Madarassy G: Analysis of CSF shunting procedure requirement in children with posterior fossa tumors. Childs Nerv Syst 19:332-336, 2003

4. Cochrane DD, Kestle JRW: The influence of surgical operative experience on the duration of first ventriculoperitoneal shunt function and infection. Pediatr Neurosurg 38:295301, 2003

5. Culley DJ, Berger MS, Shaw D, Geyer R: An analysis of factors determining the need for ventriculoperitoneal shunts after posterior fossa tumor surgery in children. Neurosurgery 34:402-408, 1994

6. Depreitere B, Dasi N, Rutka J, Dirks P, Drake J: Endoscopic biopsy for intraventricular tumors in children. J Neurosurg 106 (5 Suppl):340-346, 2007

7. Dias MS, Albright AL: Management of hydrocephalus complicating childhood posterior fossa tumors. Pediatr Neurosci 15:283-290, 1989

8. Drake JM: Endoscopic third ventriculostomy in pediatric patients: the Canadian experience. Neurosurgery 60:881-886, 2007

9. El Beltagy MA, Kamal HM, Taha H, Awad M, El Khateeb $\mathrm{N}$ : Endoscopic third ventriculostomy before tumor surgery in children with posterior fossa tumors, CCHE experience. Childs Nerv Syst 26:1699-1704, 2010

10. El-Ghandour NMF: Endoscopic third ventriculostomy versus ventriculoperitoneal shunt in the treatment of obstructive hydrocephalus due to posterior fossa tumors in children. Childs Nerv Syst 27:117-126, 2011

11. Foreman P, McClugage S III, Naftel R, Griessenauer CJ, Ditty BJ, Agee BS, et al: Validation and modification of a predictive model of postresection hydrocephalus in pediatric patients with posterior fossa tumors. J Neurosurg Pediatr 12:220-226, 2013

12. Gopalakrishnan CV, Dhakoji A, Menon G, Nair S: Factors predicting the need for cerebrospinal fluid diversion following posterior fossa tumor surgery in children. Pediatr Neurosurg 48:93-101, 2012

13. Grunert P, Charalampaki P, Hopf N, Filippi R: The role of third ventriculostomy in the management of obstructive hydrocephalus. Minim Invasive Neurosurg 46:16-21, 2003

14. Harris PA, Taylor R, Thielke R, Payne J, Gonzalez N, Conde JG: Research electronic data capture (REDCap) - a metadata-driven methodology and workflow process for providing translational research informatics support. J Biomed Inform 42:377-381, 2009

15. Klimo P Jr, Goumnerova LC: Endoscopic third ventriculocisternostomy for brainstem tumors. J Neurosurg 105 (4 Suppl):271-274, 2006

16. Kulkarni AV, Drake JM, Kestle JRW, Mallucci CL, Sgouros S, Constantini S: Endoscopic third ventriculostomy vs ce- 
rebrospinal fluid shunt in the treatment of hydrocephalus in children: a propensity score-adjusted analysis. Neurosurgery 67:588-593, 2010

17. Kulkarni AV, Drake JM, Mallucci CL, Sgouros S, Roth J, Constantini S: Endoscopic third ventriculostomy in the treatment of childhood hydrocephalus. J Pediatr 155:254-259, 259.e1, 2009

18. Lin CT, Riva-Cambrin JK: Management of posterior fossa tumors and hydrocephalus in children: a review. Childs Nerv Syst 31:1781-1789, 2015

19. Moher D, Shamseer L, Clarke M, Ghersi D, Liberati A, Petticrew M, et al: Preferred reporting items for systematic review and meta-analysis protocols (PRISMA-P) 2015 statement. Syst Rev 4:1-9, 2015

20. Morelli D, Pirotte B, Lubansu A, Detemmerman D, Aeby A, Fricx C, et al: Persistent hydrocephalus after early surgical management of posterior fossa tumors in children: is routine preoperative endoscopic third ventriculostomy justified? J Neurosurg 103 (3 Suppl):247-252, 2005

21. Raimondi AJ, Tomita T: Hydrocephalus and infratentorial tumors. Incidence, clinical picture, and treatment. J Neurosurg 55:174-182, 1981

22. Ray P, Jallo GI, Kim RYH, Kim BS, Wilson S, Kothbauer $\mathrm{K}$, et al: Endoscopic third ventriculostomy for tumor-related hydrocephalus in a pediatric population. Neurosurg Focus 19(6): E8, 2005

23. Riva-Cambrin J, Detsky AS, Lamberti-Pasculli M, Sargent MA, Armstrong D, Moineddin R, et al: Predicting postresection hydrocephalus in pediatric patients with posterior fossa tumors. J Neurosurg Pediatr 3:378-385, 2009

24. Roujeau T, Di Rocco F, Dufour C, Bourdeaut F, Puget S, Rose $\mathrm{CS}$, et al: Shall we treat hydrocephalus associated to brain stem glioma in children? Childs Nerv Syst 27:1735-1739, 2011

25. Ruggiero C, Cinalli G, Spennato P, Aliberti F, Cianciulli E, Trischitta V, et al: Endoscopic third ventriculostomy in the treatment of hydrocephalus in posterior fossa tumors in children. Childs Nerv Syst 20:828-833, 2004

26. Sainte-Rose C, Cinalli G, Roux FE, Maixner R, Chumas PD, Mansour M, et al: Management of hydrocephalus in pediatric patients with posterior fossa tumors: the role of endoscopic third ventriculostomy. J Neurosurg 95:791-797, 2001

27. Schneider C, Ramaswamy V, Kulkarni AV, Rutka JT, Remke M, Tabori U, et al: Clinical implications of medulloblastoma subgroups: incidence of CSF diversion surgery. J Neurosurg Pediatr 15:236-242, 2015

28. Takahashi Y: Long-term outcome and neurologic development after endoscopic third ventriculostomy versus shunting during infancy. Childs Nerv Syst 22:1591-1602, 2006

29. Tamburrini G, Pettorini BL, Massimi L, Caldarelli M, Di Rocco C: Endoscopic third ventriculostomy: the best option in the treatment of persistent hydrocephalus after posterior cranial fossa tumour removal? Childs Nerv Syst 24:14051412, 2008

30. Taylor WA, Todd NV, Leighton SE: CSF drainage in patients with posterior fossa tumours. Acta Neurochir (Wien) 117:16, 1992

\section{Disclosures}

The authors report no conflict of interest concerning the materials or methods used in this study or the findings specified in this paper.

\section{Author Contributions}

Conception and design: Dewan. Acquisition of data: Dewan, Lim. Analysis and interpretation of data: Dewan. Drafting the article: Dewan. Critically revising the article: all authors. Reviewed submitted version of manuscript: Dewan, Shannon, Wellons. Approved the final version of the manuscript on behalf of all authors: Dewan. Statistical analysis: Dewan. Administrative/technical/material support: Shannon. Study supervision: Wellons.

\section{Correspondence}

Michael C. Dewan, Department of Neurological Surgery, Vanderbilt University Medical Center, T-4224 Medical Center North, Nashville, TN 37232-2380. email: michael.dewan@ vanderbilt.edu. 